\title{
Reconstruction of phase shifts as functions of energy using bound-state energies and low-energy scattering data
}

\author{
I. A. Ivanov \\ Institute of Spectroscopy, Academy of Sciences of Russia, 142190, Troitsk, Russia
}

(Received 11 November 2002; published 12 March 2003)

\begin{abstract}
We propose a converging algorithm allowing to reconstruct phase shifts in a broad range of momenta using low-energy scattering data and information about bound states (if supported by the potential).

DOI: 10.1103/PhysRevA.67.032704

PACS number(s): 03.65.Nk, 02.60.-x, 34.50.-s, 34.80.-i
\end{abstract}

\section{INTRODUCTION}

A well-known tool used for describing low-energy scattering is effective range (ER) [1], expansion, which in the most often used form with first two terms retained, reads for $s$ wave

$$
k \cot \delta_{0}(k)=\frac{1}{a}+\frac{1}{2} r_{e} k^{2}
$$

Equation (1) describes small energy behavior of the phase shifts in the simplest case of only short-range interactions present. If any long-range interactions are present (such as polarization interaction) right-hand side of the Eq. (1) should be modified. For the polarization interaction corresponding formulas were given in Ref. [2]. The coefficients in these expansions can be determined either from calculations or experiment.

These expansions provide a basis for the description of the low-energy scattering for a variety of physical problems, such as electron-atom or positron-atom scattering. To better reproduce experimentally observed cross sections additional terms are often added to the low-energy expansions. Functional form of these additional terms is usually chosen so as to reproduce analytical form of high-order terms of lowenergy expansions for the phase shifts. Coefficients play the role of fitting parameters. Often used is the so-called modified effective range expansion (MERT) [3], relying on four or five fitting parameters to reproduce available low-energy scattering data.

This approach was successfully used to describe lowenergy electron scattering on neon [4], helium and argon $[5,6]$, kripton $[7,8]$. The issues related to the question as to what is the energy range where different modifications of MERT represent the phase shifts correctly, has been addressed in the paper [9]. The general conclusion was that for the electron-rare-gas atom system MERT represents phase shifts accurately up to the energies of $\approx 1 \mathrm{eV}$.

As an input information both ER or different variants of MERT rely on information about the phase shifts obtained either from theoretical calculation or experiment. In the present paper, we propose a procedure which constructs approximations to the phase shifts in a broad interval of energies and which can use as an input information both available information about scattering phase shifts and about bound states (if there are any, of course). That coefficients of low-energy expansions, at least the leading order ones, are affected by the presence of bound states is, of course, a wellknown fact. Thus, in the simplest case of expansion (1), the scattering length can be estimated if potential supports a shallow bound state. Upon this observation different extrapolation schemes in the spirit of those described in Ref. [10] and allowing to obtain information about the positive-energy spectrum using available information about the bound states can be constructed.

The procedure we are proposing attempts to achieve something more than extrapolation or fitting. In theory, the procedure described below provides a rigorous framework allowing to build a converging algorithm of constructing approximations to the phase shifts for a single-channel scattering. As an input information the proposed procedure uses a set of known phase shifts and a set of known bound states (if potential supports any). In practice, as we shall see, the proposed procedure allows to construct reliable approximations for the phase shifts for a broad region of momenta provided one or two phase shifts at low energies and energies of one or two bound states are known. Important feature of the proposed algorithm is that bound and scattering states are treated on equal footing.

\section{THEORY}

In the case of potential scattering we consider in the present paper, objects most useful for our purpose are the Jost functions. We remind very briefly [11] the basic properties of these functions. Below, for simplicity we consider only the $s$-wave scattering, all the results being directly applicable for $l>0$ without any modifications. If for the radial Schrödinger equation

$$
\psi^{\prime \prime}(r)+\left[k^{2}-2 V(r)\right] \psi(r),
$$

the regular solution satisfying $\psi(0)=0, \psi^{\prime}(0)=1$, and the pair of solutions $f_{1}(k, r)$ and $f_{2}(k, r)$ satisfying $\lim _{r \rightarrow \infty} f_{1}(k, r) e^{-i k r}=1$ and $\lim _{r \rightarrow \infty} f_{2}(k, r) e^{i k r}=1$, respectively, are found, then the pair of Jost functions $f^{+}(k)$ and $f^{-}(k)$ is defined as $\psi(r)=(1 / 2 i k)\left[f^{+}(k) f_{2}(k, r)\right.$ $\left.+f^{-}(k) f_{1}(k, r)\right]$. It follows from the boundary conditions imposed on the pair of solutions $f_{1}(k, r), f_{2}(k, r)$ that $f^{+}(k)$ and $f^{-}(k)$ are regular analytic functions of $k$ in upper and lower halves of $k$-complex plane, respectively. Moreover, $\lim _{|k| \rightarrow \infty} f^{ \pm}(k)=1$. The functions $f^{+}(k)$ and $f^{-}(k)$ are connected through $f^{-*}\left(k^{*}\right)=f^{+}(k)$, therefore only one of them 
needs consideration. Below we shall consider the function $f^{+}(k)$ analytic and regular in the upper half of the $k$-complex plane. If potential $V(r)$ supports bound states with energies $E_{n}=-\left(\kappa^{2} / 2\right), f^{+}(k)$ has simple zeros at the points $k=i \kappa$, $\kappa>0$. These properties are fairly general. As to the analytic properties of $f^{+}(k)$ in the lower half of complex $k$ plane, they depend crucially upon the properties of the potential $V(r)$. In the simplest case of the finite range $V(r)$ [i.e., $V(r)=0$ for all $r>R] f^{+}(k)$ is an entire function of variable $k$. Representation of this entire function as an infinite product leads to useful representation for the $S$ matrix [11]. For more realistic potentials, even rapidly decaying with $r$ [e.g., exponentially decaying $V(r)], f^{+}(k)$ may have singularities in the lower half of the complex $k$ plane, therefore, neither $f^{+}(k)$ nor $S$ matrix allow simple representations.

However, the fact of analycity of $f^{+}(k)$ in the upper half of the complex $k$ plane, and the fact that $f^{+}(k) \rightarrow 1$ when $|k| \rightarrow \infty$ in the upper half of the complex plane, can be usefully exploited further than it is usually done. Consider the function $h(z)$ which is analytic and regular for $\operatorname{Re}(z)>0$ and tends to zero uniformly when $z \rightarrow \infty$ in the half plane $\operatorname{Re}(z)$ $>0$. These conditions are sufficient to ensure that $h(z)$ can be represented as Laplace transform of a certain function $g(t)$. This statement when applied to $f^{+}(k)$ leads to the following representation of this function:

$$
f^{+}(k)=1+\int_{0}^{\infty} g(t) e^{i k t} d t .
$$

To ensure the condition $f^{+*}(k)=f^{+}(-k)$ which Jost function must satisfy on the real axis $g(t)$ must be real. Formula (3) is valid everywhere in the half plane $\operatorname{Im}(k)>0$. On the real axis this equation can be regarded as Fourier transform of $f^{+}(k)-1$. Inverting this transform and shifting contour of integration in the upper half plane it is easy to see that $g(t)$ is zero for $t<0$ as in Eq. (3). If $f^{+}-1$ is square integrable on the real axis, then $g(t)$ is also square integrable function and can therefore be expanded on a suitable basis set in $L^{2}$.

On the real axis $f^{+}(k)=|f(k)| e^{-i \delta(k)}$, where $\delta(k)$ is phase shift. It follows from Eq. (3) that

$$
\tan \delta(k)=-\frac{\int_{0}^{\infty} g(t) \sin k t d t}{1+\int_{0}^{\infty} g(t) \cos k t d t} .
$$

If potential supports bound state with energy $E_{n}$ $=-\kappa_{n}^{2} / 2$ then $f^{+}\left(i \kappa_{n}\right)=0$ and

$$
\int_{0}^{\infty} g(t) e^{-\kappa_{n} t} d t=-1
$$

On the basis of the Eqs. (4) and (5) a procedure allowing to construct approximations to the function $g(t)$ and hence approximations of the phase shifts can be devised as follows.

Suppose that for a given potential in Eq. (2) a set of phase shifts $\delta\left(k_{i}\right), i=1, \ldots, N_{c}$ for a set of momenta $k_{i}$ is known (either from experiment or calculation). If Eq. (2) has dis- crete spectrum suppose also that some of the discrete spectrum energies and hence a set of $\kappa_{n}, n=1, \ldots, N_{b}$ are known. Then expanding $g(t)$ on a suitable basis set in $L^{2}$,

$$
g(t)=\sum_{n=0}^{\infty} g_{n} \phi_{n}(t)
$$

Eqs. (4) and (5) can be rewritten as (summation over repeated indexes is understood)

$$
\begin{gathered}
{\left[s_{i j}+\tan \delta\left(k_{i}\right) c_{i j}\right] g_{j}=-\tan \delta\left(k_{i}\right), \quad i=1, \ldots, N_{c},} \\
e_{n j} g_{j}=-1, \quad n=1, \ldots, N_{b},
\end{gathered}
$$

and

$$
\begin{gathered}
s_{i j}=\int \sin \left(k_{i} t\right) \phi_{j}(t) d t, \quad c_{i j}=\int \cos \left(k_{i} t\right) \phi_{j}(t) d t, \\
e_{n j}=\int \exp \left(-\kappa_{n} t\right) \phi_{j}(t) d t .
\end{gathered}
$$

Retaining the first $N_{c}+N_{b}$ terms of the expansion (6), one obtains s set of $N_{c}+N_{b}$ linear equations for the first $N_{c}$ $+N_{b}$ coefficients $g_{n}$. Once the linear system for the first $N_{c}+N_{b}$ coefficients $g_{n}$ is solved, phase shifts can be found with the help of the Eq. (4).

An important feature of this procedure is that known bound states and phase shifts are treated on equal footing. Known bound states provide, thus, additional information which, as we shall see, may allow to find phase shifts for momenta lying well outside the set of momenta $k_{i}$ for which phase shifts are known. Since bound-states energies are usually obtained much easier than the phase shifts this feature of the proposed procedure may turn out quite useful. That this procedure actually works is shown in the following section.

\section{NUMERICAL RESULTS}

In this section, we consider a few examples showing applications of the outlined above procedure. The procedure is applicable for short- and long-range interactions. We shall consider both of them. In all the examples considered below we use the orthonormal set,

$$
\phi_{n}(t)=\sqrt{2 \mu} L_{n}(2 \mu t) e^{-\mu t},
$$

of Laguerre functions [12] as the basis in the expansion (6). The parameter $\mu$ is a free parameter. A few words should be said about choice of this parameter. Numerical experiments for different potentials show that the role of $\mu$ is twofold. First, it affects the rate at which the series (6) converges. Second, the system (7) degenerates quickly for some values of $\mu$. In practice, we choose $\mu$ so as to satisfy the requirement that linear system (7) should be well defined (i.e., its determinant has a value far from zero).

\section{A. Short-range interaction}

$$
\text { 1. } V(r)=-e^{-r}
$$

$s$-wave scattering in this potential allows analytic treatment [13]. This potential supports a bound state $E=-\kappa^{2} / 2$ 


\section{$V(r)=-e^{-r}$}

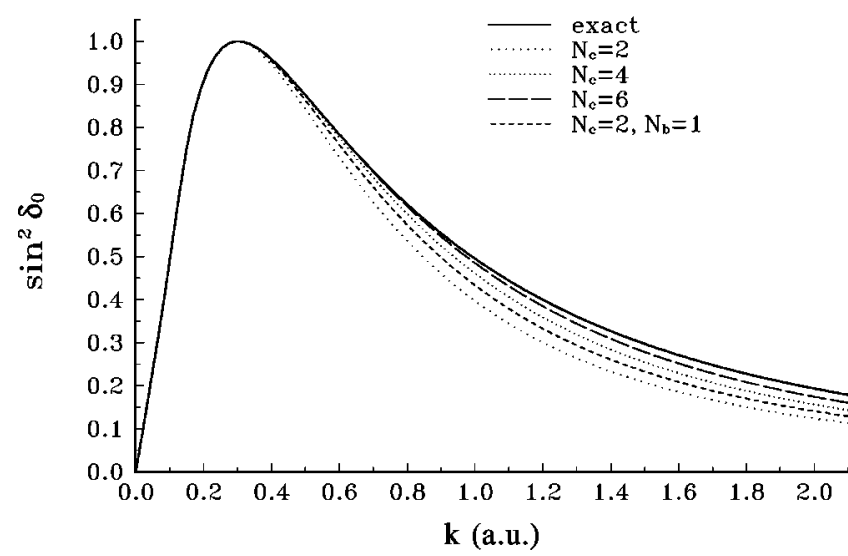

FIG. 1. Exact phase shifts and different approximations for the $-e^{-r}$ - potential ( $s$ wave).

with $\kappa \approx 0.141211308209 \ldots$. As a set of known phase shifts the phase shifts given by analytic formula from Ref. [13] for a set of momenta $k_{i}=0.01+0.05(i-1), \quad i$ $=1, \ldots, N_{c}$ were used.

This information was used as an input for constructing approximations to the function $g(t)$ following outlined above procedure based on the linear system (7).

We found that the convergence of the procedure was the fastest if one put $\mu=1 / 2$ in the formula (8). This value of $\mu$ could be expected. Indeed, inverting Fourier transform (3) and shifting contour of integration downwards in the lower half plane, one finds easily that large- $t$ behavior of $g(t)$ is determined by the nearest to the real axis singular point of $f^{+}(k)$ in the lower half plane. For the case of the $e^{-r}$ potential this singular point lies at $k=-i / 2$ [11], leading to the $e^{-t / 2}$ large- $t$ behavior of the function $g(t)$ in the formula (3).

Figure 1 shows results of different approximations constructed using first two exact phase shifts for $k=0.01$ and $k$ $=0.06\left(N_{c}=2\right)$, first four exact phase shifts for $k=0.01$ $+0.05(i-1), i=1, \ldots, 4,\left(N_{c}=4\right)$ first six exact phase shifts for $k=0.01+0.05(i-1), i=1, \ldots, 6\left(N_{c}=6\right)$, and finally first two exact phase shifts for $k=0.01+0.05(i-1)$, $i=1, \ldots, 2$ and the bound state $\left(N_{c}=2, N_{b}=1\right)$.

Approximations $N_{c}=4$ and $N_{c}=6$ are of less practical importance than $N_{c}=2$ and $N_{c}=2, N_{b}=1$ and are presented merely to illustrate the convergence of the procedure. As can be seen both $N_{c}=4$ and $N_{c}=6$ approximate phase shifts quite adequately for all $k \in[0,2]$. Taking into account that they are based on the exact phase shifts computed for $k$ not greater than $0.21\left(N_{c}=4\right)$ or $0.31\left(N_{c}=6\right)$ the overall convergence of the procedure with increasing $N_{c}$ could be considered quite satisfactory.

As another illustration of the convergence of the present algorithm, we present in Table I the values which linear system (7) gives for the coefficients of the expansion (6). The first four columns of Table I give coefficients $g_{n}$ for the four approximations listed above. The last column presents coefficient $g_{n}$ of the expansion (6) for the approximation using the first $N_{c}=8$ exact phase shifts for $k_{i}=0.01+0.05(i-1)$, $i=1, \ldots, 8$. As one can see, the coefficients $g_{i}$, especially $g_{0}$ and $g_{1}$ clearly exhibit convergence, thus confirming overall consistency of the procedure. Moreover, as one can see, the coefficients $g_{n}$ for a given approximation decay rapidly with $n$, indicating that expansion (6) converges quite rapidly. It is this latter fact which helps to understand why even the low-order approximations, such as $N_{c}=2$ on the Fig. 1 represent satisfactorily exact phase shifts in a broad range of momenta.

Of more practical importance are approximations $N_{c}=2$ and $N_{c}=2, N_{b}=1$. Indeed two exact phase shifts computed for very small momenta provide essentially the same information as first two terms of the effective range expansion. As one can see, inclusion of a bound state (approximation $N_{c}$ $=1, N_{b}=1$ ) improves the quality of the approximation $N_{c}$ $=2$ based on two low-energy phase shifts considerably. This improvement has been obtained without adding additional scattering data. One might say that this improvement has been achieved at virtually no cost since calculation of the energy of the bound state is much simpler than scattering calculation. One is tempted, therefore, to keep $N_{c}$ as low as possible, increasing $N_{b}$. Figure 2 illustrates this strategy for stronger exponential potential supporting larger number of bound states.

\section{2. $V(r)=-10 e^{-r}$}

For this potential system (7) is better defined for larger values of $\mu$. The value $\mu=3$ was used in the calculations.

This potential supports larger number of the bound states than the one considered in the preceding section. In our cal-

TABLE I. Coefficients $g_{n}$ of the expansion (6) for different approximations constructed for $-e^{-r}$ potential ( $s$ wave)

\begin{tabular}{lcccc}
\hline \hline$N_{c}=2$ & $N_{c}=2, N_{b}=1$ & $N_{c}=4$ & $N_{c}=6$ & $N_{c}=8$ \\
\hline-0.6736567 & -0.6878113 & -0.6977244 & -0.7074583 & -0.7121132 \\
-0.0609205 & -0.0898825 & -0.1140079 & -0.1390282 & -0.1516257 \\
& -0.0117970 & -0.0246498 & -0.0422330 & -0.0527445 \\
& & -0.0029783 & -0.0106895 & -0.0171528 \\
& & -0.0019719 & -0.0049347 \\
& & -0.0002011 & -0.0011667 \\
& & & -0.0002009 \\
& & & -0.0000194 \\
\hline \hline
\end{tabular}




\section{$\mathrm{V}(\mathrm{r})=-10 \mathrm{e}^{-\mathrm{r}}$}

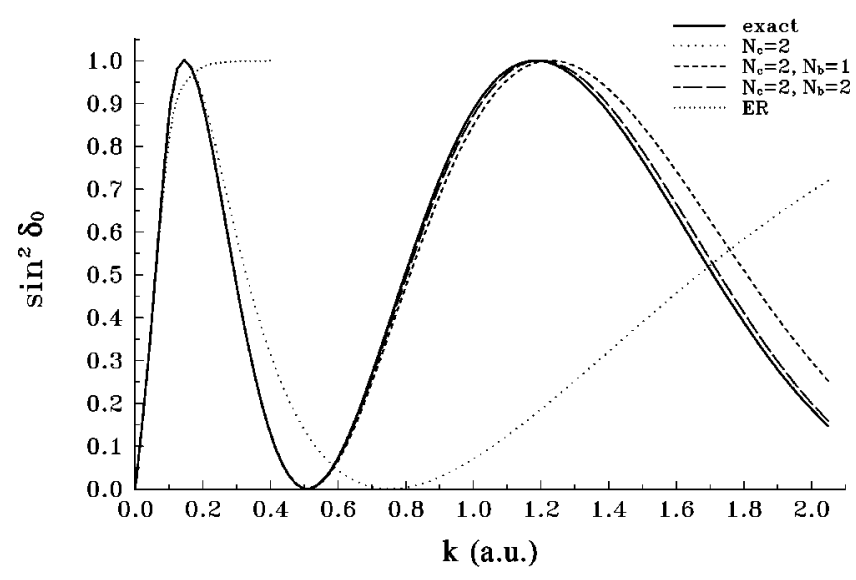

FIG. 2. Exact phase shifts and different approximations for the $-10 e^{-r}$ - potential ( $s$ wave).

culations, we used two bound states: $\kappa_{1} \approx 0.093244388$, $\kappa_{2} \approx 1.19399366$.

Figure 2 presents the results of the following approximations: $N_{c}=2$, exact phase shifts for $k=0.1, k=0.15$ were used as an input for the system (7), $N_{c}=2, N_{b}=1$ (the same phase shifts as before and bound state with $\kappa_{1}$ were used), and $N_{c}=2, N_{b}=2$ (phase shifts as before and two bound states $\kappa_{1}, \kappa_{2}$ ). Figure 2 clearly demonstrates that account of the bound states dramatically improves quality of the approximation. Account of two bound states gives quite satisfactory representation of the $s$-wave phase shifts for $k$ as large as 2 a.u.

The results given by two terms ER expansion for this potential are also shown [ER expansion for this potential is $\tan \delta_{0}(k) \approx-14.64 k-631.9 k^{3}$, using the value for $\tan \delta_{0}(k)$ given by this expansion, $\sin ^{2} \delta_{0}$ was computed and plotted]. Our simplest approximation $N_{c}=2$ using virtually the same information as the ER expansion (two phase shifts at low energies) does considerably better job at reproducing the correct phase shifts. This circumstance can be explained by the fact that representation (3) automatically guarantees that $\tan \delta(k) \rightarrow 0$ when $k \rightarrow \infty$, a condition which is, of course absent in the ER expansion.

We are by no means confined to use only low-energy phase shifts as an input for the linear system (7). Figure 3 illustrates what happens if we construct the same approximations as above with the difference that two exact phase shifts are taken from the region of larger energies $\left(k_{1}=0.4\right.$ a.u. and $k=0.65$ a.u.). It can be seen that the simplest approximation $N_{c}=2$ using only these two phase shifts does a very poor job at representing the exact phase shifts. Introducing the bound states changes the situation dramatically. Here, as in the previous example, account of the bound states leads to great improvement in the quality of approximation.

Our procedure relies on fairly general properties of the Jost function (primarily analicity in the upper half-plane) which are valid for both short-range and long-range interactions and any value of angular momentum. In the following

\section{$\mathrm{V}(\mathrm{r})=-10 \mathrm{e}^{-\mathrm{r}}$}

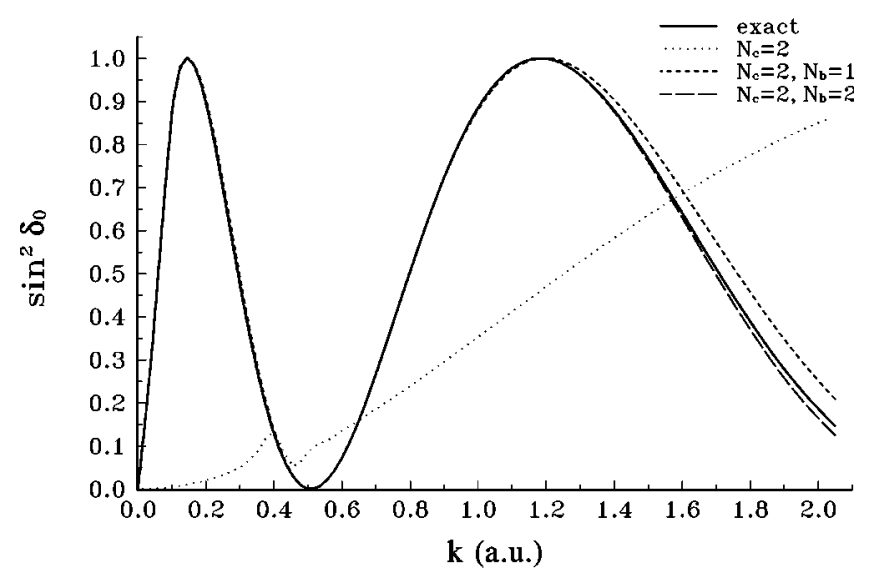

FIG. 3. Exact phase shifts and different approximations for the $-10 e^{-r}$ - potential constructed with the input phase shifts lying in the region of higher energies ( $s$ wave).

section, we consider an example of $p$-wave scattering in the presence of long-range forces.

\section{B. Long-range interaction}

$$
\text { 1. } V(r)=-8.5 e^{-r}-1 / 2\left(r^{2}+1\right)^{2}
$$

We consider in this section $p$-wave scattering for the potential which is a combination of short-range interaction and the long-range polarization interaction. This potential supports a few bound states. In constructing our approximations we shall use the bound state with $\kappa \approx 0.14152875$ (energy of the bound states and $p$-wave phase shifts reported below were found by means of numerical integration). For the parameter $\mu$ in the formula (8), we used the value $\mu=1 / 2$. For this value of $\mu$ the linear system (7) is far from degeneracy and can be easily treated numerically.

We constructed a set of approximations following exactly the same procedure as above in the case of short-range interaction: $N_{c}=2$ (uses numerically computed $p$-wave phase shifts for $k=0.05, k=0.1$ ) $, N_{c}=2, N_{b}=1$ (uses the same set of phase shifts and the bound state), $N_{c}=4$ [phase shifts for $k=0.05+0.05(i-1), i=1, \ldots, 4]$ and $N_{c}=6$ [phase shifts for $k=0.05+0.05(i-1), i=1, \ldots, 6$ were used].

In Table II the values of the coefficients $g_{n}$ obtained as a result of the numerical solution of the system (7) for this set

TABLE II. Coefficients $g_{n}$ of the expansion (6) for long-range interaction ( $p$ wave).

\begin{tabular}{lccc}
\hline \hline$N_{c}=2$ & $N_{c}=2, N_{b}=1$ & $N_{c}=4$ & $N_{c}=6$ \\
\hline-0.8101454 & -0.8168623 & -0.8247228 & -0.8159431 \\
-0.2696980 & -0.3347658 & -0.3576295 & -0.3291100 \\
& -0.0375544 & -0.0564662 & -0.0259384 \\
& & -0.0048872 & 0.0109997 \\
& & & 0.0047030 \\
& & & 0.0006341 \\
\hline \hline
\end{tabular}




\section{$\mathrm{V}(\mathrm{r})=-8.5 \mathrm{e}^{-\mathrm{r}}-0.5 /\left(\mathrm{r}^{2}+1\right)^{2}$}

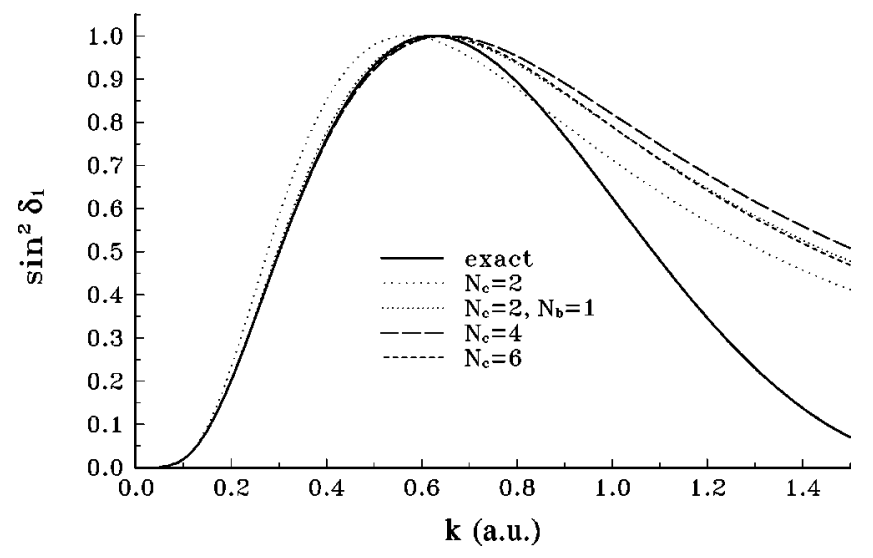

FIG. 4. Exact phase shifts and different approximations for the potential having long-range tail ( $p$ wave).

of approximations are presented. One can see that, as in the case of short-range interaction, the $g_{n}$ 's (especially the loworder ones) exhibit, first, near constancy for different approximations, and, second, decay rapidly for a given approximation, thus confirming overall validity of the approach.

A few words should be said about behavior of $g(t)$ in the case when long-range interactions are present. For the longrange interactions $f^{+}(k)$ generally has a singular point at $k$ $=0$ (in the case of short-range interactions the nearest to the real axis singularity lies in the lower half plane). This fact alters large- $t$ behavior of $g(t)$. Indeed, inverting the Fourier transform (3) one can show that in this case large- $t$ behavior of $g(t)$ is determined by the singular point at $k=0$, which generally leads to only powerlike decay of $g(t)$ for large $t$. Therefore, for long-range forces, $g(t)$, though remaining square integrable [square-integrability follows from the assumed square integrability of $\left.f^{+}(k)-1\right]$, decays more slowly than in the case of short-range interaction.

The Fig. 4 shows the phase shifts produced by the listed above set of approximations and "exact" phase shifts computed numerically. As in the examples of the short-range interactions considered above one can see that account of a bound state turns out to be more important than adding more phase shifts at positive energies. Indeed the $N_{c}=2, N_{b}=1$ approximation does a fairly good job at describing phase shifts for $k$ as large as 0.8 and gives results virtually identical to the results given by the approximation $N_{c}=8$ using far greater number of points. One observes, that in the present case of long-range forces the quality of approximations for larger $k$ ( $k \approx 1$ a.u. and larger) is not so good as in the examples of purely short-range interaction. This may be due to the mentioned above property of only powerlike decay of $g(t)$ for large $t$. One could try to cope with this problem [e.g., by introducing square integrable but only powerlike decaying terms in Eq. (6)], but this issue lies beyond the scope of the present paper.

Finally, we consider two more realistic examples of electron-hydrogen and positronium-positronium scattering.
TABLE III. Phase shifts (calculated and results of different approximations constructed in the framework of the present procedure) for electron-hydrogen scattering.

\begin{tabular}{lcccc}
\hline \hline$k$ & Ref. [14] & $N_{c}=2$ & $N_{c}=1, N_{b}=1$ & $N_{c}=3$ \\
\hline 0.1 & 2.5535 & 2.5535 & 2.5535 & 2.5535 \\
0.2 & 2.0668 & 2.0668 & 2.0678 & 2.0668 \\
0.3 & 1.6966 & 1.6873 & 1.6900 & 1.6966 \\
0.4 & 1.4154 & 1.3843 & 1.3884 & 1.4158 \\
0.5 & 1.2009 & 1.1329 & 1.1381 & 1.1967 \\
0.6 & 1.0409 & 0.9177 & 0.9237 & 1.0193 \\
0.7 & 0.9307 & 0.7292 & 0.7358 & 0.8701 \\
0.8 & 0.8874 & 0.5616 & 0.5684 & 0.7405 \\
\hline \hline
\end{tabular}

Strictly speaking (2) in these cases is rather an integrodifferential than differential equation. However, our procedure relies upon fairly general properties of the Jost functions, which may be assumed to remain valid for integrodifferential equations.

\section{2. e-H scattering}

We consider $s$-wave $e-H$ scattering in the singlet spin state. The accurate values of the phase shifts were taken from Ref. [14] (these values for the phase shifts were confirmed by different workers, e.g., $[15,16])$. The system has one bound state with binding energy 0.0277501 a.u., correspondingly $\kappa \approx 0.235585$.

Following without any modifications the usual procedure, we construct a set of approximations [the value $\mu=3$ was used in Eq. (8)]: $N_{c}=2$ (uses the calculated phase shifts for $k=0.1, k=0.2$ ), $N_{c}=1, N_{b}=1$ (uses phase shift for $k=0.1$ and the bound state), and $N_{c}=3$ (uses the calculated phase shifts for $k=0.1, k=0.2, k=0.3$ ). As one can see all these approximations do fairly good job at reproducing phase shifts (Table III). The approximation $N_{c}=1, N_{b}=1$ is particularly interesting (and perhaps most important). This approximation uses only one result of scattering calculation proper (phase shift at $k=0.1$ a.u.). The second ingredient, the energy of the bound state is obtained quite easily by means of any standard procedure of calculating bound-state energies. At the same time the error of this approximation is less than $10 \%$ for $k$ less 0.6 a.u.

Our second realistic example is $P s-P s$ scattering.

\section{Ps-Ps scattering}

We consider $s$-wave scattering, with zero total spin of the system. The calculations of the phase shifts performed in the papers [17-19] produced virtually coinciding results. We shall use the results given in Ref. [18] as an input in constructing the approximations. The system has a bound state with the binding energy 0.0160037 a.u. [20], corresponding $\kappa \approx 0.178906$ a.u. We choose $\mu=3$ in the Eq. (8).

The following approximations were constructed: $N_{c}=2$ (uses the calculated phase shifts for $k=0.1, k=0.15$ ), $N_{c}$ $=1, N_{b}=1$ (uses phase shift for $k=0.1$ and the bound state), and $N_{c}=3$ (uses the calculated phase shifts for $k=0.1, k$ $=0.15, k=0.2$ ). Figure 5 shows the results. Again, of most 


\section{Ps-Ps scattering}

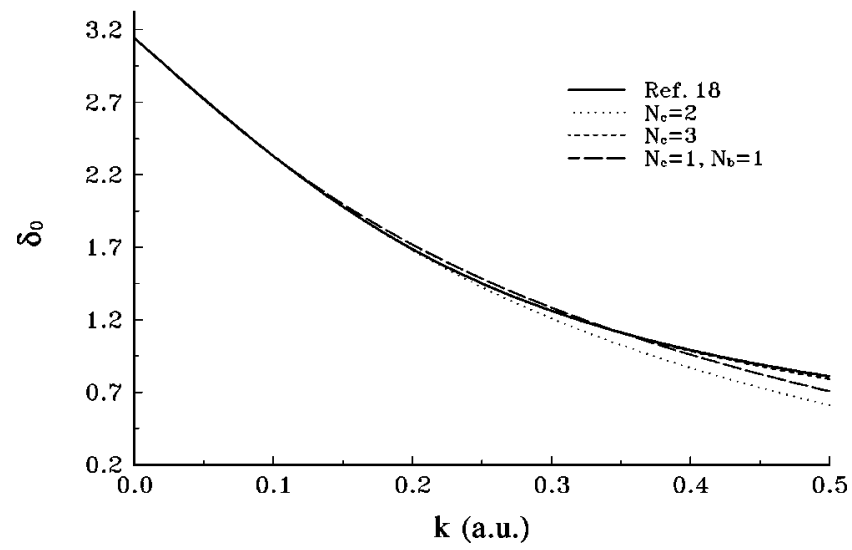

FIG. 5. Exact phase shifts and different approximations for the $P s-P s$ scattering ( $s$ wave).

practical importance is the approximation $N_{c}=1, N_{b}=1$, which uses just one result of scattering calculation (phase shift at $k=0.1$ ) and which can be seen to do a fairly good job at reproducing calculated phase shifts.

As far as applications of the present procedure to the case of Coulomb forces are concerned, all the basis ingredients leading to the formulas (4) and (5) remain valid. Therefore, in theory, the present procedure could be applied to the case of Coulomb forces as well. However, one should expect that in this case the convergence of the procedure will be rather slow. Indeed, any good approximation to the Jost function in this case should reproduce a series of zeros, the true Jost function posseses, converging to $k=0$ along the imaginary axis. This is rather a demanding task for an approximation of low order.

As a final test of the proposed procedure, we consider in the following section simple model system exhibiting resonance behavior.

\section{Resonances}

We consider a very simple model system-square- well potential: $V(r)=-1$ for $r<3, V(r)=0$ otherwise. Both phase shifts for any angular momentum and eigenenergies can easily be found with the help of known analytic formulas [13]. We shall consider $p$-wave case. For $L=1$ the potential supports a few bound states, for the one we shall use in our approximations $\kappa \approx 0.78324195$ a.u.

As in all previous examples, we construct the following set of approximations $N_{c}=2$ (uses the calculated phase shifts for $k=0.05, k=0.1), N_{c}=2, N_{b}=1$ (the same phase shifts and the bound state), $N_{c}=4, N_{b}=1$ (phase shifts for $k$ $=0.05, k=0.1, k=0.15, k=0.2$ and the bound state).

As an illustration of the features of the procedure, we did not use before, we constructed one more approximation which used phase shifts for $k=0.05, k=0.1, k=0.15$ and the bound state and which, moreover, was designed to reproduce correct Born limit. In the framework of the present procedure this can be done fairly easily. Indeed, inspection of the for-

\section{$V(r)=-\theta(3-r)$}

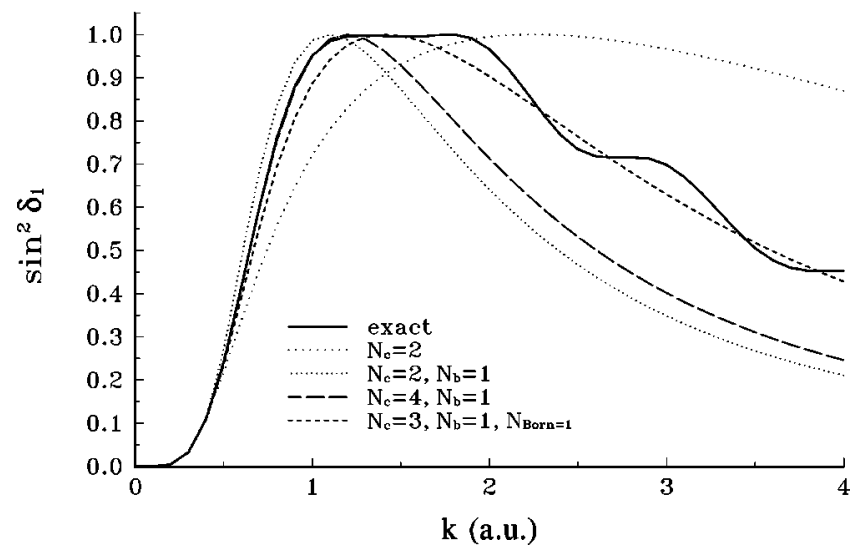

FIG. 6. Exact phase shifts and different approximations for square-well potential ( $p$ wave).

mulas (4) shows that Born limit is determined by the small$t$ behavior of the function $g(t)$. In particular, if asymptotically, for very large momenta, one has $\tan \delta(k)$ $\rightarrow \tan \delta_{B o h r}(k)$, where $\tan \delta_{B o h r}(k)=A / k$, then to reproduce this behavior, $g(t)$ in the Eq. (4) should satisfy $g(0)=-A$, which can be verified easily by means of standard technique of asymptotic evaluation of integrals. It is easy to show that for the potential we consider $\tan \delta_{B o h r}(k)=3 / k$. Therefore, to reproduce large- $k$ limit correctly we add one more equation to the system (7) imposing requirement $g(0)=-3$. For the approximation thus constructed, we use the notation $N_{c}$ $=3, N_{b}=1, N_{\text {Born }}=1$, symbolic notation $N_{\text {Born }}=1$ signifies that equation $g(0)=-3$ was added to the system (7). Figure 6 presents the results the approximations described above give for the phase shifts. One can see again, that inclusion of the bound state leads to the considerable improvement of the approximation. For $k>2$ a.u. exact phase shift exhibit rather complicated oscillatory behavior. Approximations of a rather low order which we used could not possibly reproduce such fine details for large $k$. Still, approximation $N_{c}=3, N_{b}$ $=1, N_{\text {Born }}=1$, which has correct Born limit built in, reproduces general features of the phase shifts behavior quite satisfactory.

\section{CONCLUSIONS}

That Jost function is uniquely determined provided the phase shifts are given for all $k$ and the bound states (if any) are known, is, of course, a well-known fact [13]. The present paper attempts to devise a procedure allowing to reconstruct the Jost function and hence the phase shifts provided some information about scattering or bound states of Eq. (2) is available. Several examples considered above show that a few low-energy phase shifts and a few bound states allow to reproduce correct phase shifts in a rather broad interval of momenta.

Theoretical foundation of this approach is provided by the general statement cited above. A few words should be said about issues of purely numerical character. 
Generally, the system (7) degenerates rather quickly when order of approximation increases. For example, the data in the Table I contain results of the approximation $N_{c}=8$ for the exponential potential. Further increase of the order of approximation (e.g., attempt to determine $g_{n}$ 's for the $N_{c}$ $=10$ approximation) caused problems of numerical character due to high degree of degeneracy of the system (7). This problem can be partly tackled with by choosing the value of $\mu$ in Eq. (8) carefully. The strategy might be to choose $\mu$ so as to maximize the determinant of the system (7) for a given low-order approximation. Alternatively, since this degeneracy arises when phase shifts at closely spaced point $k_{i}$ are taken as an input for the outlined above procedure, taking phase shifts at more widely separated points may remedy the situation.

Actually, this problem of near degeneracy of the system (7) hardly arises in practice and does not pose any serious problems in practical applications of the present procedure since this effect manifests itself only if one attempts to construct approximations of large order. In practical applications the most interesting and the most important approximations are the low-order ones $\left(N_{c}, N_{b}\right.$ with $\left.N_{c}, N_{b}=0,1,2,3\right)$. For such low-order approximations, of course, no numerical problems of the character described above can arise.

A natural question arises how can one be sure that a given approximation yields good representation of the phase shifts, if only a few low-energy phase shifts are known. The strategy, which our experience with model and real physical systems suggests is valid, is to construct a sequence of approximations, e.g., $N_{c}=1, N_{b}=1, N_{c}=2, N_{b}=1$. If the leading order coefficients $g_{n}$ exhibit stability (akin to the stability of $g_{n}$ 's in Tables I and II), both $N_{c}=1, N_{b}=1$ and $N_{c}=2, N_{b}$ $=1$ can be expected to give good results in representing the phase shifts.

To conclude, we propose a procedure allowing to incorporate available information about scattering and bound states of a given system and use it as in input to represent phase shifts quite accurately in a region of momenta considerably broader than the region in which input phase shifts lie. Additional information making such a representation possible is provided by the known bound states. Account of the bound states in the framework of the present procedure may lead to substantial improvement in representing the correct phase shifts as functions of energy.
[1] G.F. Chew and M.L. Goldberger, Phys. Rev. 75, 1637 (1949).

[2] T.F. O'Malley, L. Rosenberg, and L. Spruch, Phys. Rev. 125, 1300 (1962).

[3] T.F. O'Malley, Phys. Rev. 130, 1020 (1963).

[4] T.F. O’Malley and R.W. Crompton, J. Phys. B 13, 3451 (1980).

[5] D.E. Golden, Phys. Rev. 151, 48 (1966).

[6] G.N. Haddad and T.F. O’Malley, Aust. J. Phys. 35, 35 (1982).

[7] S.J. Buckman and B. Lohmann, J. Phys. B 19, 2457 (1986).

[8] S.J. Buckman and B. Lohmann, J. Phys. B 20, 2457 (1987).

[9] S.J. Buckman and J. Mitroy, J. Phys. B 22, 1365 (1989).

[10] M.J. Seaton, Rep. Prog. Phys. 46, 167 (1983).

[11] R.G. Newton, Scattering Theory of Waves and Particles (Springer-Verlag, New York, 1982).

[12] Handbook of Mathematical Functions, edited by M. Abramovitz and I.A. Stegun (Dover, New York, 1967).
[13] N.F. Mott and H.S.W. Messy, The Theory of Atomic Collisions (Clarendon Press, Oxford, 1965).

[14] C. Schwartz, Phys. Rev. 124, 1468 (1961).

[15] I. Shimamura, J. Phys. Soc. Jpn. 30, 1702 (1971).

[16] O.I. Tolstikhin, V.N. Ostrovsky, and H. Nakamura, Phys. Rev. Lett. 79, 2026 (1997).

[17] I.A. Ivanov, J. Mitroy, and K. Varga, Phys. Rev. Lett. 87, 063201 (2001).

[18] I.A. Ivanov, J. Mitroy, and K. Varga, Phys. Rev. A 65, 022704 (2002).

[19] J. Shumway and D.M. Ceperley, Phys. Rev. B 63, 165209 (2001).

[20] Y. Suzuki and K. Varga, Stochastic Variational Approach to Quantum-Mechanical Few-Body Problems (Springer, New York, 1998). 